\title{
Inversely designed, 3D-printed personalized template-guided interstitial brachytherapy for vaginal tumors
}

\author{
Shuhei Sekii, MD!, Kayoko Tsujino, MD, PhD!, Kengo Kosaka, PhD², Satoshi Yamaguchi, MD, PhD³, Hikaru Kubota, MD! \\ Yoko Matsumoto, MD', Yosuke Ota, MD', Ryohei Sasaki, MD, PhD4, Toshinori Soejima, MD, PhDl \\ 'Department of Radiation Oncology, Hyogo Cancer Center, Akashi, Hyogo, ${ }^{2}$ Department of Radiology, Hyogo Cancer Center, Akashi, \\ Hyogo, ${ }^{3}$ Department of Gynecologic Oncology. Hyogo Cancer Center, Akashi, Hyogo, ${ }^{4}$ Division of Radiation Oncology, Kobe University \\ Graduate School of Medicine, Kobe, Hyogo, Japan
}

\begin{abstract}
Purpose: In this paper, we report cases of two patients with vaginal tumor who underwent interstitial brachytherapy (ISBT), using three-dimensional (3D)-printed personalized templates designed inversely from computed tomography (CT) or magnetic resonance (MR) images.

Material and methods: Patient 1 presenting with vaginal vault recurrence was planned to receive whole pelvis external beam radiotherapy (EBRT) followed by ISBT. The tumor invaded the paracolpium; thus, we planned to administer ISBT to include the tumor and vaginal membrane. A template was designed with holes for plastic needle applicator insertion considering the appropriate direction based on pre-treatment medical images. Patient 2 presenting with vaginal cancer was scheduled to receive EBRT and ISBT because of a paracolpium invasion. Before ISBT, MR imaging was performed with vaginal cylinder inserted in the patient's vagina. By measuring the length of the tumor manually and projecting the tumor orthogonally to a plane parallel to the bottom surface of the cylinder applicator, a template was designed. Computer-aided design software was used for planning both templates. Polycarbonate/ acrylonitrile-butadiene-styrene resin was selected as material of the templates.

Results: Patient 1 received 4-fraction ISBT one week apart. A mean of 10 applicators were inserted through the holes of the template in an average of 9 minutes (range, 5-15 minutes). All applicators were inserted toward the planned directions. Median minimum dose covering $90 \%\left(\mathrm{D}_{90 \%}\right)$ of the clinical target volume (CTV) was 634 cGy. Patient 2 underwent three-fraction irradiation twice daily at 6-hour interval. All applicators were inserted through the inside of the template. The median $\mathrm{D}_{90 \%}$ of the CTV was 703 cGy. No grade 3 or higher toxicity were found in both series.

Conclusions: 3D-printed templates designed using medical images are useful, especially for ISBT of vaginal tumors. Further verification of clinical indications, design of templates, and manufacturing process are needed.

Key words: 3D-printing, brachytherapy, vaginal tumor.

\section{Purpose}

Three-dimensional (3D) printing techniques are widely used in medical fields. In radiation oncology, this technique offers patient-individualized uniformly thick boluses for electron radiotherapy [1] and phantoms for radiotherapy dosimetry [2]. In the field of brachytherapy, some studies on the usage of 3D-printing techniques have been reported. Harris and colleagues examined the feasibility of 3D-printing mold [3]. Huang et al. reported on individual 3D-printed templates using preplanned data about needle pathways and ${ }^{125}$ I seeds positions for lowdose-rate brachytherapy of head and neck cancers [4].
Jones et al. concluded that 3D-printing applicators could be used for highly conformal superficial dose distributions by using preplanned catheter orientations [5]. Other authors reported molds for brachytherapy for skin cancers created using 3D scanner [6]. Another study showed two cases of interstitial brachytherapy for vagina tumors performed using 3D-printed custom-made cylinders [7]. Therefore, the introduction of 3D-printing techniques has spread in brachytherapy.

For irradiation of cervical cancers, intracavitary brachytherapy (ICBT) with or without interstitial brachytherapy (ISBT) can be performed by using several applicators such as the Fletcher applicator, Venezia applicator (Elekta AB,

Address for correspondence: Shuhei Sekii, MD, Department of Radiation Oncology, Hyogo Cancer Center, Received: 03.05.2018 13-70 Kitaojicho, Akashi, Hyogo 673-8558, Japan, phone: +81 78-929-1151, fax: +81 78-929-2380, 
Stockholm, Sweden), and Manchester-style applicator (Varian Medical System Inc., Palo Alto, CA, USA). On the other hand, applicators for the treatment of vaginal tumors, including vaginal cancer or vaginal recurrence of gynecological cancer, are scarce. Patients with tumors $\leq 5 \mathrm{~mm}$ thick are treated with ICBT using cylinders such as vaginal cylinder applicators (Elekta AB, Stockholm, Sweden), which are also commercially available. When administering ISBT in patients with tumors $>5 \mathrm{~mm}$ thick, insertion of needle applicators of perineum or vagina is needed. Although commercially available perineal templates such as Syed/Neblett GYN template (Best Medical International, Inc., Springfield, VA, USA) are present, it is frequently time-consuming procedure to adjust the inserted needle applicators because of little flexibility of insertion position in these templates. Thus, a high threshold still exists for beginners of ISBT administration.

Here, we presented two cases of patients who underwent brachytherapy using personalized, 3D-printed templates designed from computed tomography (CT) or magnetic resonance (MR) images.

\section{Material and methods}

\section{Patient 1}

A 49-year-old woman with stage IB1 cervical cancer who initially underwent modified radical hysterectomy, bilateral salpingo-oophorectomy, and pelvic lymphadenectomy. Initial pathological examination revealed squamous cell carcinoma with no lymphovascular invasion, no lymph node metastases, and negative vaginal margins; thus, she had not received adjuvant treatment. Seven years later, she developed a vaginal vault recurrence with internal iliac node metastasis. She was scheduled to receive whole-pelvis external beam radiotherapy (EBRT) at a dose of $45 \mathrm{~Gy}$ in 25 fractions, with concurrent weekly administration of cisplatin, followed by brachytherapy at a dose of $24 \mathrm{~Gy}$ in four fractions weekly and boost irradiation of lymph node metastasis up to $10 \mathrm{~Gy}$ in five fractions. Before EBRT, the tumor invaded the paracolpium, but not the pelvic wall, with a size of $35 \mathrm{~mm}$. Therefore, ISBT including the tumor and vaginal membrane was planned. The vaginal size was determined using a commercially available vaginal cylinder applicator (Elekta $\mathrm{AB}$, Stockholm, Sweden). The tumor diameter and length from the vaginal vault to the tumor was measured based on pre-treatment CT or MR images (Figure 1), and the tumor was simplified into a quadrangular pyramid (Figure 2). A template was designed using computer-aided design (CAD) software (Fusion 360 v.2.0.3174, Autodesk Inc., San Francisco, CA, USA) (Figure 3). First, we designed a solid that was a combination of a cylinder and a hemisphere with holes parallel to the long axis to insert applicators. Second, holes of template were planned in oblique directions to the long axis of the solid because the tumor invaded the paracolpium. The angle was determined by inserting a line into the deepest area of the tumor from the vaginal introitus before the other inserting lines were determined to maintain an approximately $1 \mathrm{~cm}$ distance between each line in the deepest area.

\section{Patient 2}

A 67-year-old woman who underwent hysterectomy for cervical cancer developed primary vaginal cancer 21 years later. She had not received adjuvant radiotherapy and no recurrence had been observed. The clinical stage was CT2 vaginal cancer with metastasis of the right obturator lymph node (N1) and no distant metastases. She was planned to receive concurrent chemoradiotherapy consisting of weekly administration of cisplatin and EBRT including whole-pelvic radiotherapy at a dose of 50.4 Gy in 28 fractions, prophylactic inguinal nodal irra-
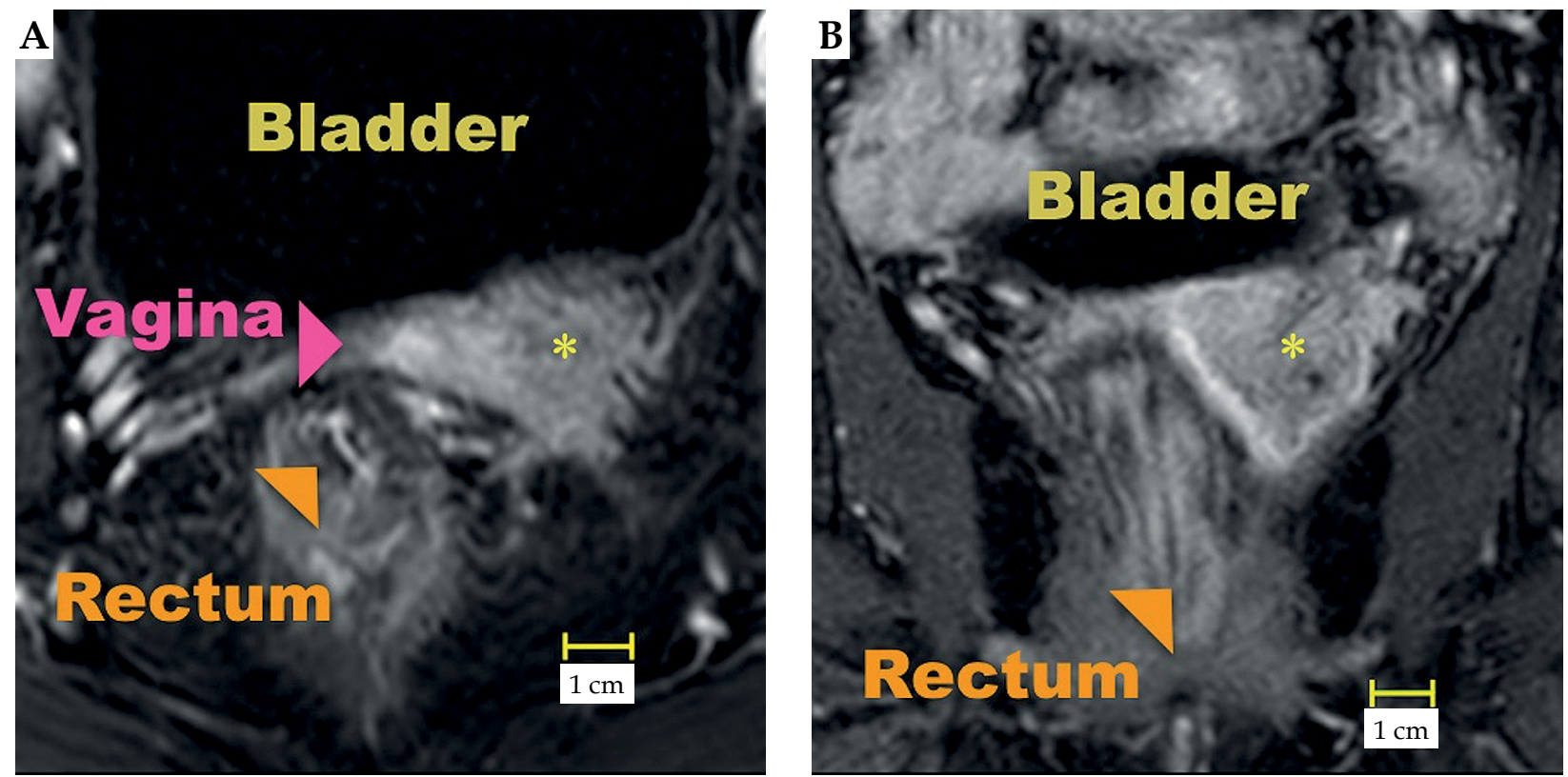

Fig. 1. Pre-treatment T1-weighted fat saturated gadolinium enhanced magnetic resonance images of Patient 1. (A) Axial view.

(B) Coronal view. Yellow asterisk represents the vaginal recurrent tumor 

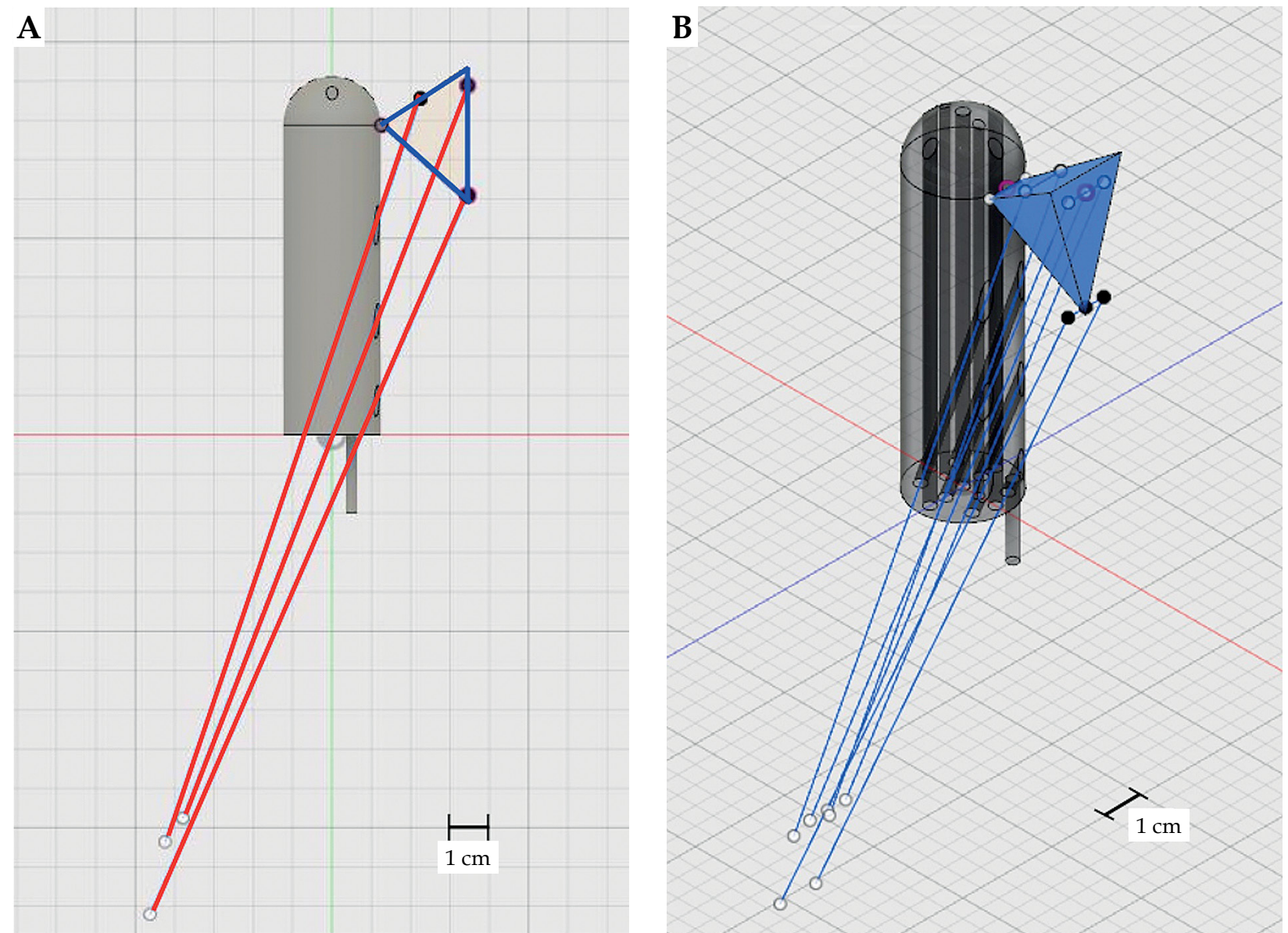

Fig. 2. Blueprint of personalized template for interstitial brachytherapy of Patient 1. (A) Gray figure is template, red lines represent $20 \mathrm{~cm}$ plastic applicators, and blue triangle shows the simplified tumor. (B) Blue semitransparent quadrangular pyramid is the simplified tumor

diation at a dose of 45 Gy in 25 fractions, boost irradiation of the lymph node metastasis up to 10 Gy in 5 fractions, and brachytherapy. Although the tumor was observed to have decreased in size two weeks before administering brachytherapy, based on MR images acquired with a vaginal cylinder applicator $25 \mathrm{~mm}$ in diameter (Elekta $\mathrm{AB}$, Stockholm, Sweden), the paracolpium invasion to the right side of the pelvis still existed (Figure 4), and ISBT was scheduled. With reference to the acquired MR images, we measured the length of the tumor manually and the tumor was orthogonally projected to an imaginary plane parallel to the bottom surface of the cylinder applicator. The template designed was a united body consisting of a rigid body by imparting a thickness of $1 \mathrm{~cm}$ to the orthogonal projection image and a $25 \mathrm{~mm}$ cylinder (Figure 5). We used the same CAD software and brachytherapy planning system as for Patient 1 .

Two and three hours were required to design the templates for Patients 1 and 2, respectively. All steps used in the design in the CAD software were completely manual. Both data of the solid were output in stereolithography (STL) format and the template was outsourced to be generated by a 3D printer (DMM.com Base, Kaga, Japan). Polycarbonate/acrylonitrile-butadiene-styrene (PC-ABS) polymer alloy was selected as the material of the template because PC possesses sterilizability and biocompatibility [8], and ABS has flexibility. The layer thickness of the printer was $127 \mu \mathrm{m}$, and the filling rate was $100 \%$. The time intervals between ordering and arrival were 6 and 7 days for Patients 1 and 2, respectively. Before treatment, the Hounsfield unit of this template was identified as approximately -50 . Treatment planning was performed using a brachytherapy planning (Oncentra, Elekta $A B$, Stockholm, Sweden). ${ }^{192}$ Ir HDR-RALS (MicroSelectron V3, Elekta AB, Stockholm, Sweden) was used for irradiation.

Our study was approved by the institutional ethical review board, and was performed in accordance with the ethical standards laid down in the 1964 Declaration of Helsinki and its later amendments.

\section{Results}

\section{Patient 1}

Before treatment, the maximum diameter of the tumor was $35 \mathrm{~mm}$. After the patient received 30.6 Gy of EBRT, MR revealed a tumor size of $28 \mathrm{~mm}$. The patient received a total of four times of ISBT with a week interval. In the first three courses of ISBT, the template (Figure 6) was inserted into the vagina, and 11 plastic applicators were inserted through the holes of the template into the tumor in 


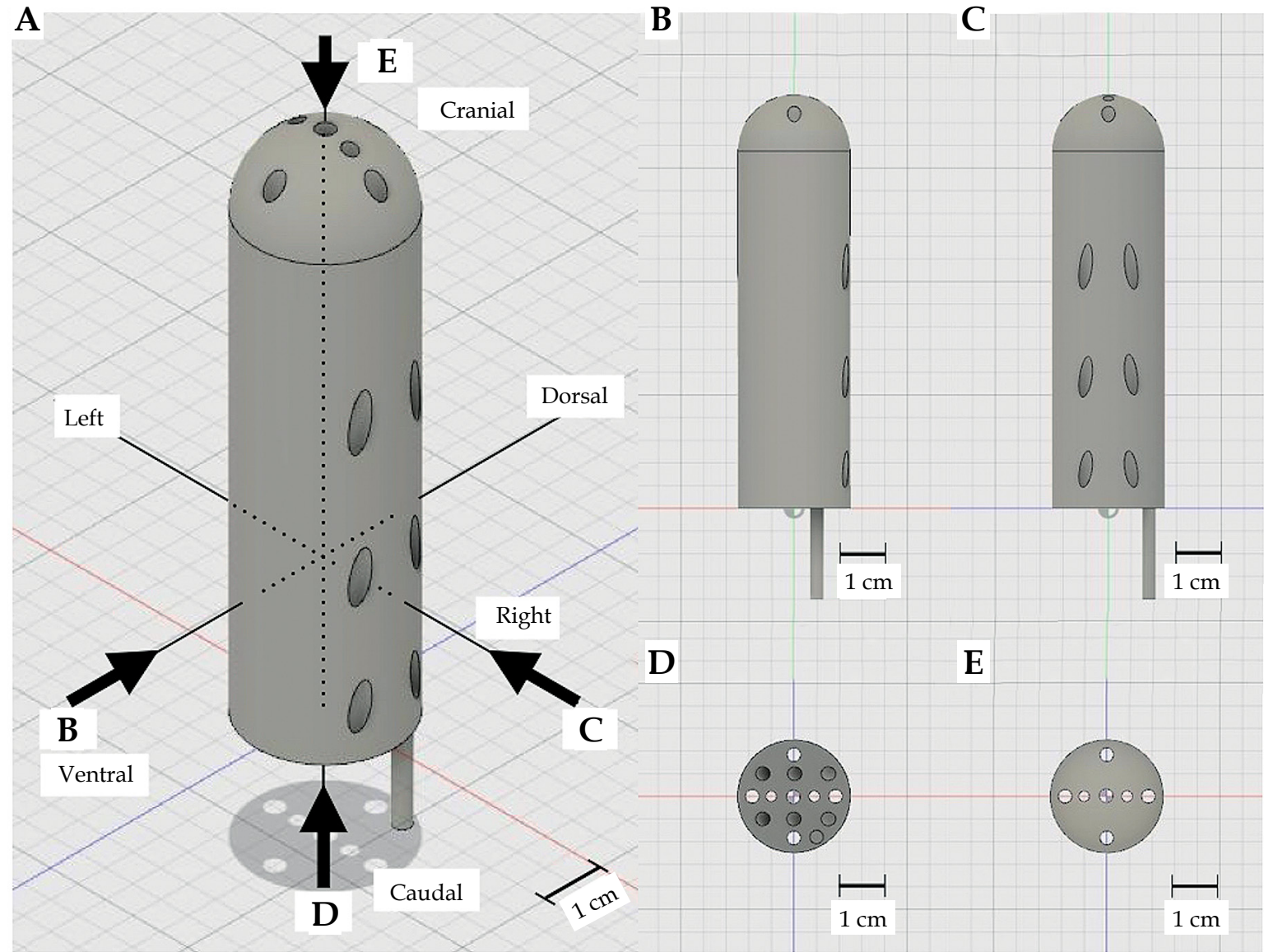

Fig. 3. Bird's eye view and projection views of the template of Patient 1. (A) Bird's eye, (B) front, (C) side, (D) bottom, and (E) top views of the template

an average of 10 minutes (range, 5-15 minutes). The clinical target volume (CTV) was defined as the volume of the tumor and whole vaginal membrane. The brachytherapy plan is depicted in Figure 7. The respective median minimum dose covering $90 \%\left(\mathrm{D}_{90 \%}\right)$ of the CTV and the volume receiving $100 \%$ dose of the prescribed dose $\left(\mathrm{V}_{100 \%}\right)$ of the CTV was $6.3 \mathrm{~Gy}$ and $94.3 \%$. In the last course of ISBT, the template was inserted into the vagina in $6 \mathrm{~min}-$ utes, 7 plastic applicators were inserted, and the CTV was defined as the volume of the tumor. $\mathrm{D}_{90 \%}$ and $\mathrm{V}_{100 \%}$ of the CTV were 7.2 Gy and $98.3 \%$, respectively. Table 1 summarizes the dose parameters of the CTV and organ at risk at each fraction. Complete response was achieved 2 months after treatment. The patient exhibited no grade 3 and higher acute side effects according to common terminology criteria for adverse events (CTCAE) version 4.0.

\section{Patient 2}

Before the treatment, the maximum diameter of the tumor was $115 \mathrm{~mm}$. After the patient received 37.8 Gy of EBRT, the tumor size was $35 \mathrm{~mm}$ (Figure 4). On the first day of ISBT, 16 plastic needles were inserted under the combination of epidural anesthesia and sedation. No applicators inserted through the outside of the designed template were needed. Because of a shortness of length of cylindrical part of the template, a cotton ball was compensated between the top of template and vaginal cuff.
ISBT was conducted twice daily at 6 hour intervals, while maintaining the patient in bed overnight throughout the treatment period. ISBT was delivered at $18 \mathrm{~Gy}$ in three fractions of 6 Gy each. Brachytherapy was planned using CT imaging on the first day and this plan was adjusted based on CT imaging performed on the following day. The CTV was defined as the tumor and whole vaginal membrane. Figure 8 shows the brachytherapy plan. The

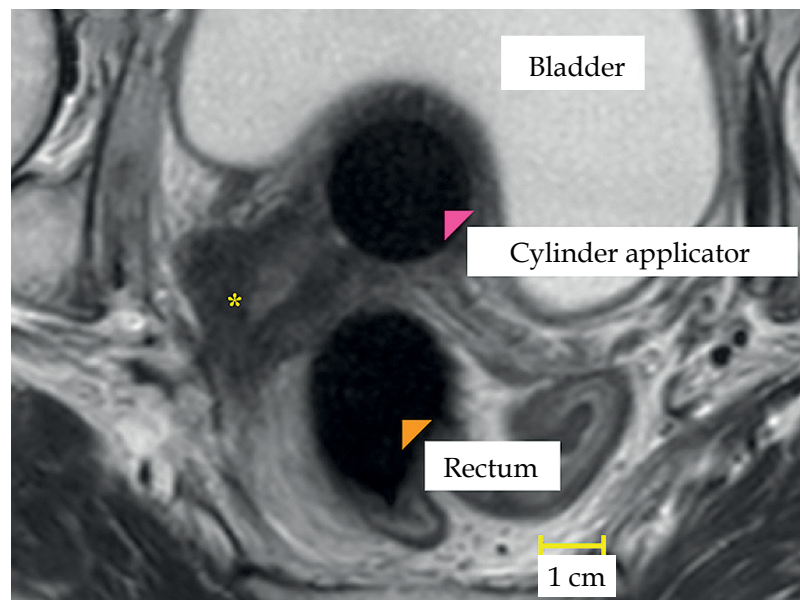

Fig. 4. Pre-brachytherapy T2-weighted magnetic resonance image of Patient 2. Yellow asterisk represents the vaginal carcinoma 

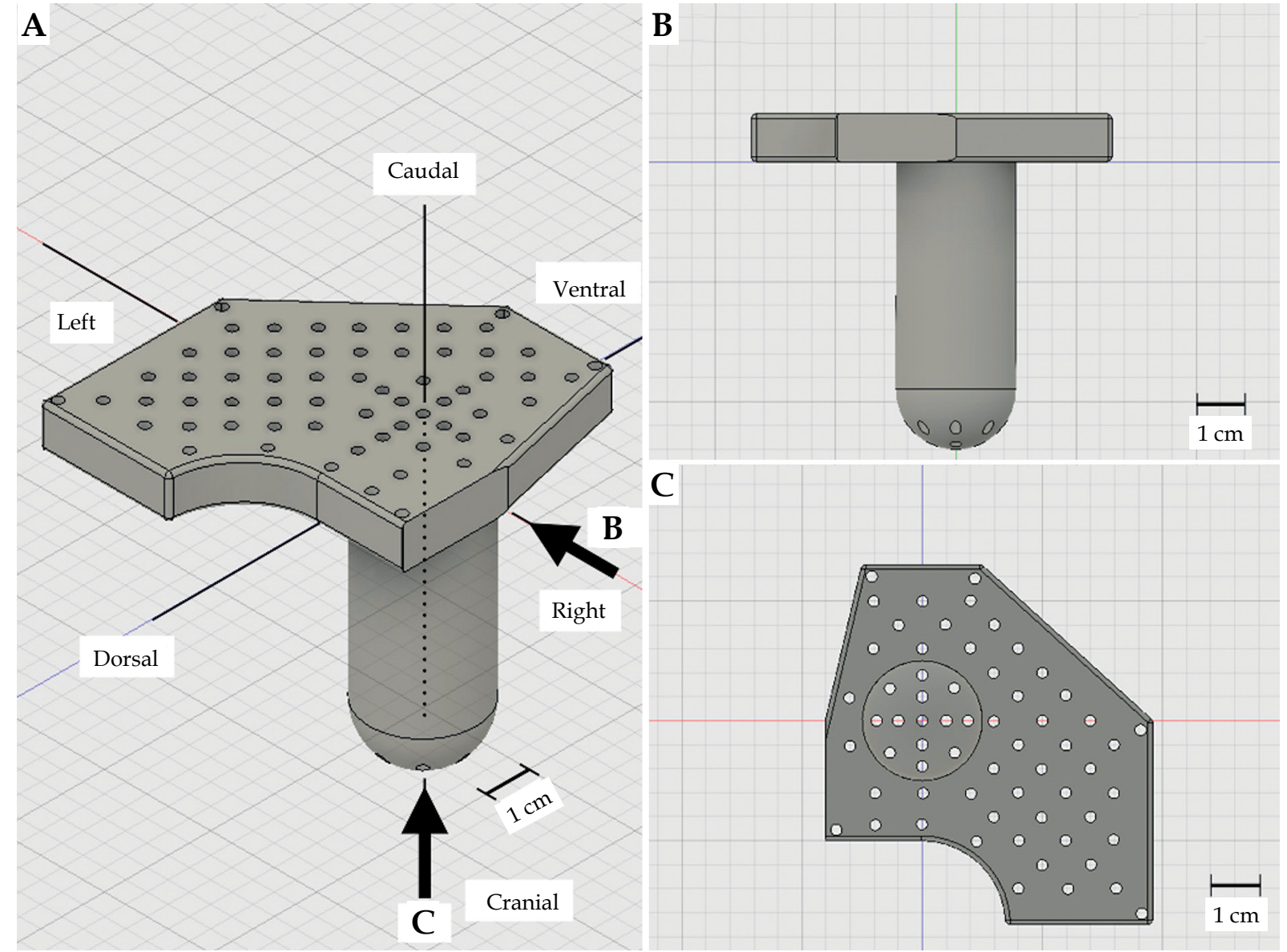

Fig. 5. Bird's eye view and projection views of the template of Patient 2. (A) Bird's eye, (B) side, and (C) top views of the template

median $\mathrm{D}_{90 \%}$ of the CTV was $7.0 \mathrm{~Gy}$ and respective median minimum doses covering $2 \mathrm{~cm}^{3}\left(\mathrm{D}_{2 \mathrm{~cm}}{ }^{3}\right)$ of organ at risk were 5.9 Gy, 4.9 Gy, 5.1 Gy, and 4.8 Gy in the bladder, rectum, sigmoid colon, and small bowel, respectively. Complete response was achieved 2 months after the treatment. The patient exhibited no severe acute side effects of treatment.

\section{Discussion}

We accomplished the generation of templates for ISBT for vaginal tumors designed inversely by using medical images. In case of Patient 1 , the inserted applicators were briefly indwelt in appropriate directions within a short period using the template, which resulted in benefits to the patient and beginners of brachytherapy administration. Because of reduced pain while inserting applicators through the vagina than through the perineum empirically, the template with oblique holes was beneficial in terms of pain under local anesthesia for patients in many Japanese facilities, which faced shortage of anesthesiologists. Beginners of brachytherapy administration could briefly insert applicators diagonally in shorter time. 3D-printing techniques can also resolve the niche problem of the lack of commercially available templates for patients with vaginal tumors. Wiebe et al. reported on intracavitary brachytherapy for vaginal recurrence. Patients who exhibited a vaginal cuff with distorted shape due to previ-

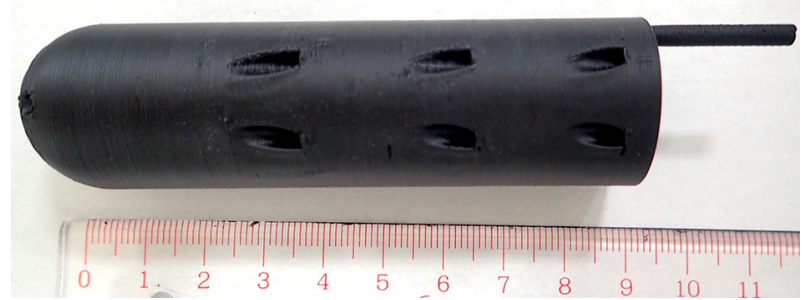

Fig. 6. Personalized template of Patient 1

ous hysterectomy generated stereolithographic intracavitary applicators based on CT images [9]. Another paper has reported the workflow of manufacturing and implementing vaginal applicators created by 3D printers. In one of two cases of interstitial brachytherapy in the paper, applicators were inserted through a 3D-printed template, and extra applicators were inserted per perineum outside the template [7]. Our study was different in regards with the design of anticipated oblique holes.

The aspect of the template being designed based on a simplified polygon or orthogonally projection by measuring the length manually in medical images, demonstrated a room for improvement. For example, we could automatically design individual templates using CAD software reading tumor data in Digital Imaging and Communications in Medicine format extracted from medical images. 

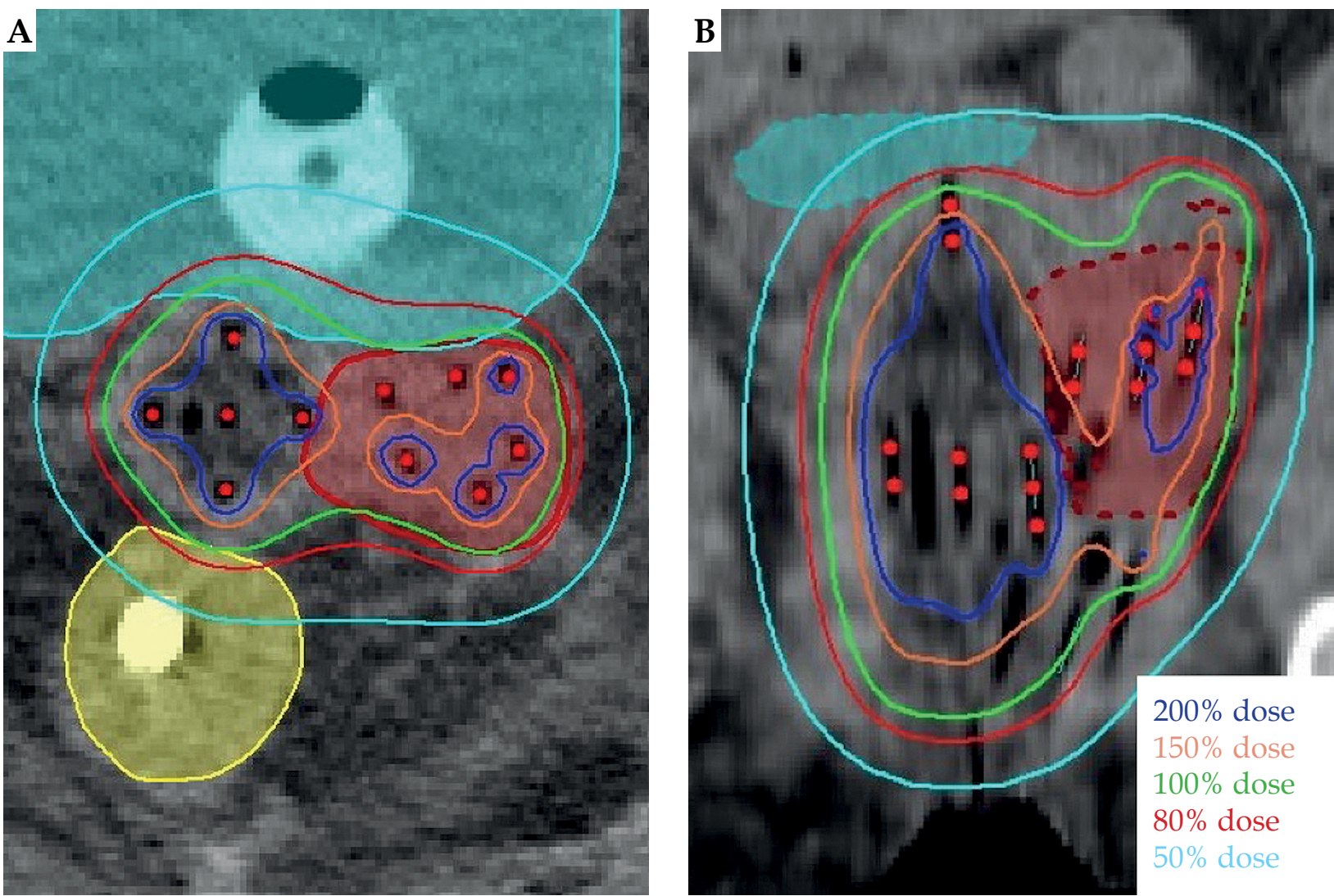

Fig. 7. Brachytherapy dose distribution of Patient 1: (A) Axial view. (B) coronal view. The template shows lower CT attenuation than for water (magenta dotted line). Thick red line in (A) and dotted line in (B) is the GTV, and the volume represents the bladder (cyan) and rectum (yellow). CT - computed tomography; GTV - gross tumor volume

Table 1. Dose parameters of clinical target volume (CTV) and organ at risk at each fraction in Patient 1

\begin{tabular}{llllll} 
& & $\# 1$ & $\# 2$ & $\# 3$ & \#4 \\
\hline CTV & $\mathrm{D}_{90 \%}(\mathrm{~Gy})$ & 6.3 & 6.4 & 6.2 & 7.2 \\
\hline Bladder & $\mathrm{D}_{2 \mathrm{~cm}}{ }^{3}(\mathrm{~Gy})$ & 5.3 & 5 & 4.9 & 2.8 \\
\hline Rectum & $\mathrm{D}_{2 \mathrm{~cm}}{ }^{3}(\mathrm{~Gy})$ & 5.8 & 5 & 4.8 & 1.2 \\
\hline Sigmoid colon & $\mathrm{D}_{2 \mathrm{~cm}^{3}}(\mathrm{~Gy})$ & 3.2 & 3.2 & 2.6 & 2.4 \\
\hline Small bowel & $\mathrm{D}_{2 \mathrm{~cm}}{ }^{3}(\mathrm{~Gy})$ & 1.7 & 1.3 & 0.8 & 0.5
\end{tabular}

$D_{90 \%}$ - minimum dose covering $90 \% ; D_{2 \mathrm{~cm}^{3}}$ - minimum dose covering $2 \mathrm{~cm}^{3}$

When semi-automatic or automatic design is implemented, less time is required to design personalized templates using the latest medical images before brachytherapy, to create the template using 3D printer. For both Patients 1 and 2, the 3D-printed templates were outsourced one week before obtaining these. The shortness of the cylindrical part of the template for Patient 2 was assumed to be caused by shrinking of the tumor between design and ISBT. Further verification of clinical indications, design of templates, and the manufacturing process are needed.

Both patients reported herein harbored vaginal tumors without uteruses due to previous hysterectomy, which was assumed to have contributed to the success of the brachytherapy using personalized templates. When personalized templates are created for multicatheter ISBT for bulky cervical cancer, it may be difficult to design the templates inversely because of the motion of the uterus. In a study by Beadle et al., the mean interfraction motions of cervical cancer were 16 and $8 \mathrm{~mm}$ in the anterior-posterior and right-left dimensions, respectively [10], whereas interfraction motions of vagina vault decreased. Rash et al. reported that the mean vaginal interfraction motions were 7 and $3 \mathrm{~mm}$ in anterior-posterior and right-left dimensions, respectively [11]. Both tumors were considered to be ideal objectives as initial inversed design and manufacturing. Further studies are mandatory to examine the design of templates for ISBT for bulky cervical cancer from appropriate images.

PC-ABS was selected as the material of the template due to its strength, heat resistance, flexibility, bio- 

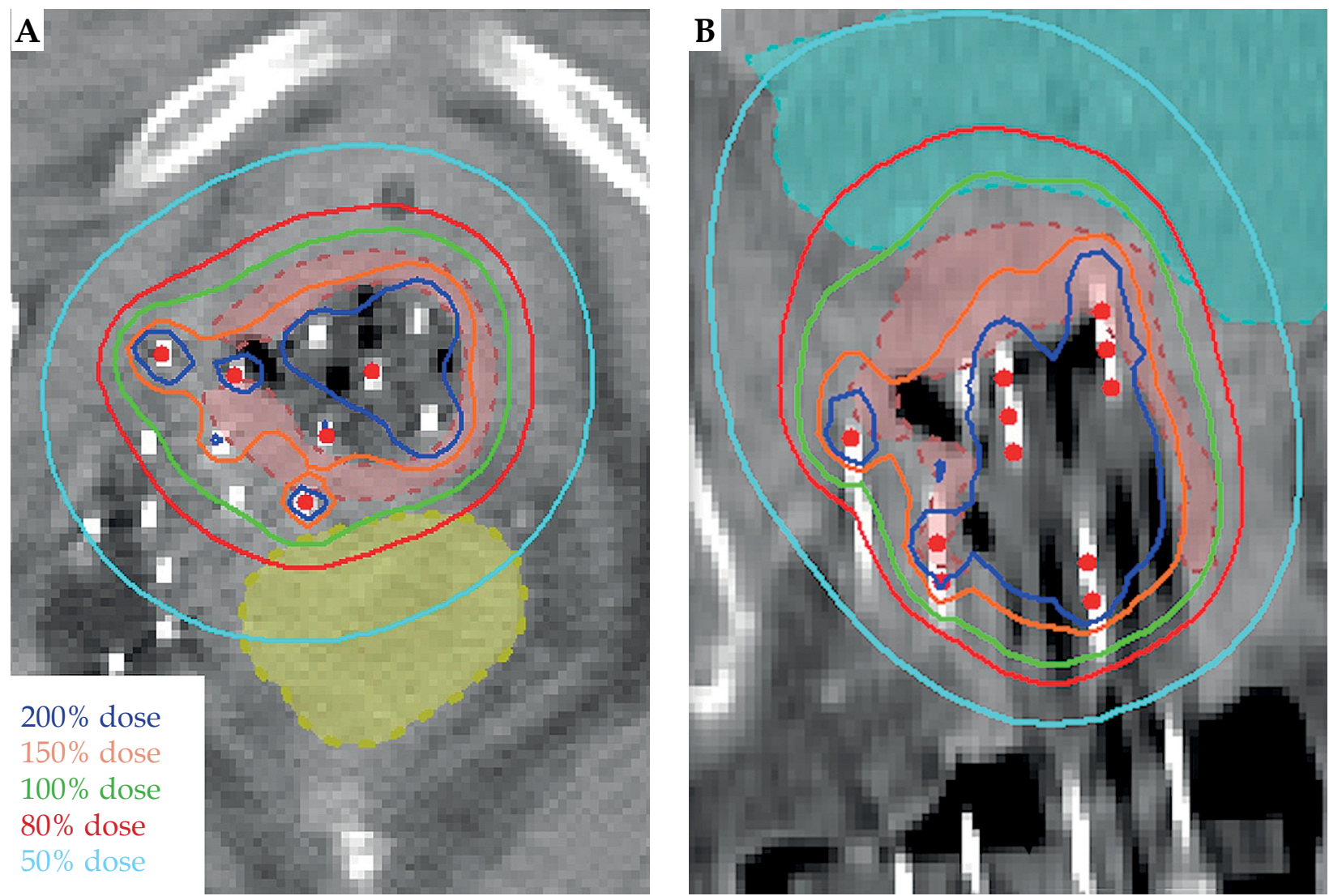

Fig. 8. Brachytherapy dose distribution of Patient 2. (A) Axial view. (B) Coronal view. Template shows lower CT attenuation than for water. Dotted line is the CTV, and the volume represents the bladder (cyan) and rectum (yellow). CT - computed tomography; CTV - clinical target volume

compatibility, certification of ISO 10993 standard, and sterilizability using ethylene oxide. These properties of PC-ABS were also attributed to being used in the automotive and electronics industries. As shown in Figure 6 , because PC-ABS has lower Hounsfield units than water, the distinction between the template and surrounding tissue was made briefly. On the other hand, some impact on dose distributions existed because our brachytherapy planning system (Oncentra) performed dose calculations without inhomogeneity correction. Cunha et al. reported the dose attenuation properties of 3D-printing products in another paper [12]. A recent study emanating from MD Anderson Cancer Center reported the time courses of change in Hounsfield units for 4 types of 3D-printed materials [13]. As they mentioned, it is mandatory to take note of the radiodensity of materials before using 3D-printed products for radiotherapy. Introducing a brachytherapy planning system that calculates with homogeneity correction would dispel concerns about dose distribution. Templates made of PC-ABS can also be used for planning, based on MR imaging due to its non-magnetic characteristic, which would contribute to the transition to MR-based gynecologic brachytherapy planning.

In our study, reference images to design the template were the pre-treatment CT and the MR images. We were aware that the tumor would reduce in size during EBRT. Ideally, we could manufacture the template by referring to medical images shortly before the first course of brachytherapy. Possessing our own 3D printer also solved this issue of reduction of tumors in part. Under such circumstances, designing and manufacturing templates early after obtaining medical images is needed. The shift from manual to semi-automatic or automatic design is also mandatory to reduce the time of treatment.

\section{Conclusions}

3D printing templates designed inversely using manual measurements on medical images have potential abilities in performing ISBT for vaginal tumors, in which few applicators or templates for ISBT for vaginal tumors may exist. Further studies are needed to alter manual design to semi-automatically or automatically design.

\section{Acknowledgements}

This study was financially supported in part by a research grant from the Japan Radiological Society from Bayer.

\section{Disclosure}

Authors report no conflict of interest. 


\section{References}

1. Lukowiak M, Jezierska K, Boehlke M et al. Utilization of a 3D printer to fabricate boluses used for electron therapy of skin lesions of the eye canthi. J Appl Clin Med Phys 2017; 18: 76-81.

2. Kairn T, Crowe SB, Markwell T. Use of 3D Printed materials as tissue-equivalent phantoms. In: Jaffray DA (ed.). World Congress on Medical Physics and Biomedical Engineering, June 7-12, 2015, Toronto, Canada. Springer International Publishing, Cham 2015: 728-731.

3. Harris BD, Nilsson S, Poole CM. A feasibility study for using ABS plastic and a low-cost 3D printer for patient-specific brachytherapy mould design. Australas Phys Eng Sci Med 2015; 38: 399-412.

4. Huang MW, Zhang JG, Zheng L et al. Accuracy evaluation of a 3D-printed individual template for needle guidance in head and neck brachytherapy. J Radiat Res 2016; 57: 662-667.

5. Jones EL, Tonino Baldion A, Thomas C et al. Introduction of novel 3D-printed superficial applicators for high-dose-rate skin brachytherapy. Brachytherapy 2017; 16: 409-414.

6. Arenas M, Sabater S, Sintas A et al. Individualized 3D scanning and printing for non-melanoma skin cancer brachytherapy: a financial study for its integration into clinical workflow. J Contemp Brachytherapy 2017; 9: 270-276.

7. Sethi R, Cunha A, Mellis K et al. Clinical applications of custom-made vaginal cylinders constructed using three-dimensional printing technology. J Contemp Brachytherapy 2016; 8: 208-214.

8. Sohn J-I, Lim ST, Park SH et al. Effect of a reactive-type flame retardant on rheological and mechanical properties of PC/ ABS blends. J Mater Sci 2003; 38: 1485-1491.

9. Wiebe $\mathrm{E}$, Easton $\mathrm{H}$, Thomas $\mathrm{G}$ et al. Customized vaginal vault brachytherapy with computed tomography imaging-derived applicator prototyping. Brachytherapy 2015; 14: 380-384.

10. Beadle BM, Jhingran A, Salehpour M et al. Cervix regression and motion during the course of external beam chemoradiation for cervical cancer. Int J Radiat Oncol Biol Phys 2009; 73: 235-241.

11. Rash D, Hagar Y, Cui J et al. Interfraction motion of the vaginal apex during postoperative intensity modulated radiation therapy: are we missing the target? Int J Gynecol Cancer 2013; 23: 385-392.

12. Cunha JA, Mellis K, Sethi R et al. Evaluation of PC-ISO for customized, 3D Printed, gynecologic 192-Ir HDR brachytherapy applicators. J Appl Clin Med Phys 2015; 16: 5168.

13. Craft DF, Kry SF, Balter P et al. Material matters: Analysis of density uncertainty in 3D printing and its consequences for radiation oncology. Med Phys 2018; 45: 1614-1621. 